\title{
Evaluation of the therapeutic effect of PUVA therapy in chronic vitiligo patients
}

\section{Kandala Bhaskara Narayana}

Professor, Dept. of Department of Dermatology, Venereology and Leprosy, MNR Medical College and Hospital, Fasalwadi, Telangana, India

*Corresponding Author:

Email: kbn.dermatology1955@gmail.com

\begin{abstract}
Introduction: Vitiligo is an acquired pigmentary disease, characterized by depigmented macules or patches which distracts the psychosocial behaviour of patients. Various therapeutic modalities have been admintered over time with variable outcome. Psoralen followed by irradiation with UV-A (PUVA) is one of the effective treatment methods for chronic vitiligo cases who had failed to react the other treatment methods.

Aim: To assess the efficacy of therapeutic effect of oral psoralen followed by ultraviolet-A irradiation (PUVA) therapy for chronic vitiligo cases.

Materials and Methods: A total 36 patients of both sexes with acral, acrofacial and generalised vitiligo with more than $20 \%$ spread over body, between age group 15-60 years were recruited. 8 MOP tablets are given with empty stomach according to body weight. All cases were undergone to UVA exposure artificial phototherapy chamber starting with a dose of $4 \mathrm{~J} / \mathrm{m}^{2}(\mathrm{Dosage}$ depends on Fitzpatrick skin type) over whole body after $90 \mathrm{~min}$. Clinical response was evaluated as marked response rate.

Results: Generalised vitiligo was seen in $66.6 \%$ cases, acral was seen in $16.6 \%$ cases, acrofacial was seen in $16.6 \%$ cases. Erythema was the common side effect followed by xerosis, pruritus, nausea and vomiting, bulla and burning. The mean duration of treatment was 14.52 months with mean no of sessions is 62.18 .

Conclusion: PUVA therapy is a well-established treatment modality for vitiligo with very minimal risk of malignancies and has less side effects like erythema and xerosis. PUVA is safe, effective, and cosmetically acceptable. Generalized vitiligo was responded well to the treatment.
\end{abstract}

Keywords: Psoralen and ultraviolet A (PUVA), Generalised vitiligo, Acral vitiligo, Acrofacial vitiligo.

\section{Introduction}

Vitiligo is one of the most common ancient diseases known to mankind characterized by depigmented macules and patches. ${ }^{1}$ It is caused by selective destruction of cutaneous melanocytes, which affects both sexes and can be developed at any age. Vitiligo has a prevalence of $0.5 \%-1 \%$, which impairs the quality of life of a sufferer. ${ }^{2}$

Vitiligo has multiple treatment modalities including psoralen and ultraviolet A (PUVA) therapy and narrow band ultraviolet $B$ (NB-UVB), topical therapy like topical corticosteroids, topical vitamin D3 analogues and topical calcineurin inhibitors and surgical therapies like split thickness graft and mini grafts, but these are often unsatisfactory. ${ }^{3}$

Psoralen followed by irradiation with UV-A (PUVA) is one of the well-established treatments for vitiligo. PUVA causes repigmentation by activating inactive melanocytes in the hair root sheath and middle and the lower part of hair follicle. ${ }^{4}$ For effective results PUVA has to be given for a prolonged duration with at least 100-200 sessions at least A days apart, 2-3 times a week. With the reference to above literature, this study was conducted to evaluate the efficacy of therapeutic effect of oral psoralen followed by ultraviolet-A irradiation (PUVA) therapy for chronic vitiligo cases.

\section{Materials and Methods}

The present prospective study was conducted in Department of Dermatology, venereology and leprosy
(DVL), MNR Medical College and Hospital during April 2016 to January 2018. A total 36 patients of both sexes between 15-60 years were recruited.

Inclusion Criteria: Cases with acral, acrofacial and generalised vitiligo with more than $20 \%$ spread over body were included.

Exclusion Criteria: Cases not willing to participate, with photosensitive skin disorders, with renal dysfunction, pregnancy and in lactation were excluded.

Informed consent was obtained from all the cases. All cases were subjected to detail clinical examination and were examined for pattern of vitiligo, area skin involved, lesions count, lesions colour and mucosal involvement. A complete haemogram and microbial analysis was conducted for regular parameters. Lesions over the body were evaluated with Rule of Nine. Based on the body weight, $8 \mathrm{MOP}$ tablets are given with empty stomach. After 90 minutes cases were allowed to UVA exposure artificial phototherapy chamber starting with a dose of $4 \mathrm{~J} / \mathrm{m}^{2}$ (Dosage depends on Fitzpatrick skin type) over whole body. Procedure was continued twice a week Started with standard initial dose of 2 $\mathrm{J} / \mathrm{cm}^{2}$ and was increased $0.5 \mathrm{~J} / \mathrm{Sq} . \mathrm{cm}$ dose depends on subject response.

Clinical response was evaluated as marked response rate, defined as $<25 \%$ is mild response, $26 \%$ $50 \%$ is moderate response, $51 \%-75 \%$ is good response and $>75 \%$ is marked response in the lesioned area. The data was tabulated and statistical analysis was done by using SPSS statistical software tool. 


\section{Results}

A total 36 cases (20 males and 16 females) with acral, acrofacial and generalised vitiligo with more than $20 \%$ spread over body were considered. Among the cases, generalised vitiligo was seen in $66.6 \%$ cases, acral was seen in $16.6 \%$ cases, acrofacial was seen in $16.6 \%$ cases. No cases with segmental and focal vitiligo in this study (Table 1).

Table 1: Clinical distribution of type of vitiligo

\begin{tabular}{|l|c|c|c|c|}
\hline \multirow{2}{*}{ Type of vitiligo } & \multicolumn{4}{|c|}{ Total number of cases (n=36) } \\
\cline { 2 - 5 } & Stable & Progressive & Number & Percentage \\
\hline Generalized vitiligo & 8 & 16 & 24 & $66.67 \%$ \\
\hline Acral vitiligo & 2 & 4 & 6 & $16.6 \%$ \\
\hline Acro facial vitiligo & 3 & 3 & 6 & $16.6 \%$ \\
\hline Segmental & - & - & - & - \\
\hline Focal & - & - & - & - \\
\hline
\end{tabular}

Table 2: Side effects by PUVA ( $n=36)$

\begin{tabular}{|l|c|c|c|c|c|c|}
\hline \multirow{2}{*}{ Symptoms } & \multicolumn{2}{|c|}{ Generalised (n=24) } & \multicolumn{2}{c|}{ Acral (n=6) } & \multicolumn{2}{c|}{ Acrofacial (n=6) } \\
\cline { 2 - 7 } & Number & Percentage & Number & Percentage & Number & Percentage \\
\hline $\begin{array}{l}\text { Nausea and } \\
\text { vomiting }\end{array}$ & 7 & $19.4 \%$ & 1 & $2.7 \%$ & 2 & $5.5 \%$ \\
\hline Erythema & 13 & $36.1 \%$ & 3 & $8.3 \%$ & 4 & $11.1 \%$ \\
\hline Bulla & 4 & $11.1 \%$ & - & - & 1 & $2.7 \%$ \\
\hline Xerosis & 9 & $25 \%$ & 2 & $5.5 \%$ & 2 & $5.5 \%$ \\
\hline Pruritus & 10 & $27.7 \%$ & 1 & $2.7 \%$ & 1 & $2.7 \%$ \\
\hline Burning & 1 & $2.7 \%$ & - & - & 1 & $2.7 \%$ \\
\hline Others & - & - & - & - & - & - \\
\hline
\end{tabular}

Acute side effects in generalised vitiligo was erythema, pruritus, xerosis followed by nausea and vomiting, bulla and burning. In acral vitiligo group, erythema, xerosis, pruritus, nausea and vomiting. In acrofacial group, erythema, xerosis, nausea and vomiting, bulla, pruritus and burning (Table 2).

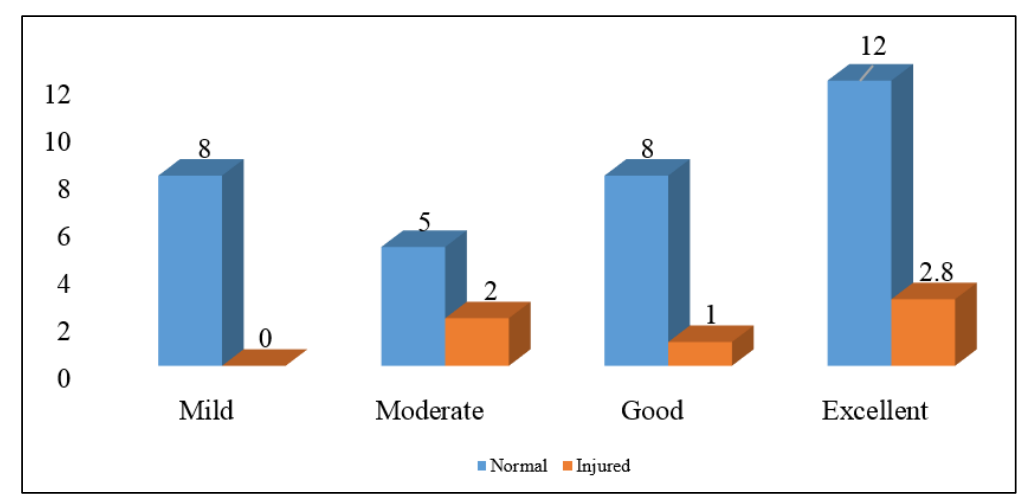

Fig. 1: Relationship between PUVA and physical and chemical injury

Table 3: Overall response of various types of vitiligo to PUVA therapy

\begin{tabular}{|l|c|c|c|c|c|}
\hline Type & $\begin{array}{c}\text { Mild response } \\
(<\mathbf{2 5 \%})\end{array}$ & $\begin{array}{c}\text { Moderate } \\
\text { response (26-50\%) }\end{array}$ & $\begin{array}{c}\text { Good response } \\
(\mathbf{5 1 - 7 5 \% )}\end{array}$ & $\begin{array}{c}\text { Marked response } \\
(>\mathbf{7 5 \%})\end{array}$ & No response \\
\hline Generalized $(\mathrm{n}=24)$ & 3 & 4 & 11 & 6 & - \\
\hline Acral $(\mathrm{n}=6)$ & - & 3 & 3 & - & - \\
\hline Acrofacial $(\mathrm{n}=6)$ & 1 & 2 & 3 & - & - \\
\hline
\end{tabular}

In generalised vitiligo, excellent response was seen in $6(25 \%)$ cases, good response in $11(45.8 \%)$ cases, moderate response in $4(16.6 \%)$ cases and mild response in $3(12.5 \%)$ cases. In acral group, good response was seen in $3(50 \%)$ cases and moderate response was seen in $3(50 \%)$ cases. In acrofacial group, good response was seen in $3(50 \%)$ cases, moderate response was seen in $2(33.3 \%)$ cases and mild response was seen in $1(16.6 \%)$ case (Table 3$)$. 
A total 12 cases had positive family history of vitiligo. Alopecia areata $(30.5 \%)$ was commonest associated autoimmune disorder followed by

hyperthyroidism (13.8\%) and hypothyroidism (11.1\%) (Fig. 2)

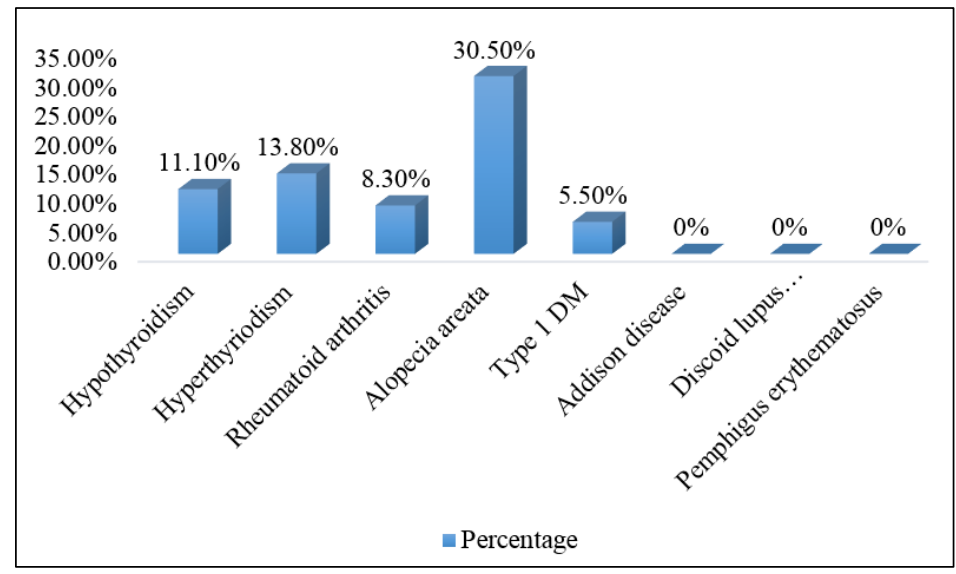

Fig. 2: Associated autoimmune disorders in vitiligo

Table 4: Overall response rate of PUVA therapy in vitiligo cases

\begin{tabular}{|l|c|}
\hline \multicolumn{1}{|c|}{ Clinical demographics of cases } & Mean \pm SD \\
\hline Age & $37.5 \pm 10.82$ \\
\hline Duration of disease (months) & $98.40 \pm 38.7$ \\
\hline$\%$ of area involved & $31.36 \pm 8.76$ \\
\hline No.of sessions & $62.18 \pm 32.54$ \\
\hline Duration of treatment & $14.52 \pm 7.98$ \\
\hline Cumulative dose $\left(\mathrm{J} / \mathrm{cm}^{2}\right)$ & $230.4 \pm 126.34$ \\
\hline Last visit & $21.86 \pm 5.72$ \\
\hline Regimentation $(50-75 \%)$ & $17(47.2 \%)$ \\
\hline
\end{tabular}
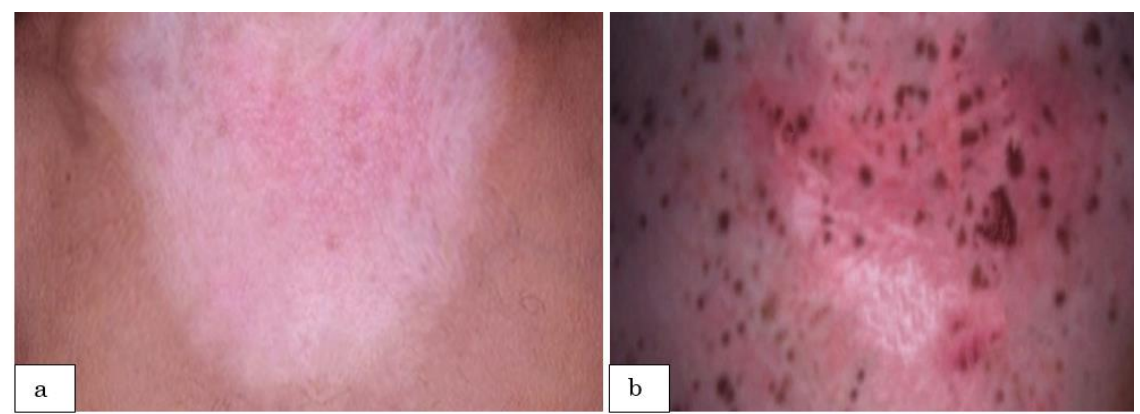

Fig. 3: Effect of PUVA therapy before and after treatment; (a): Before treatment), (b): After treatment

\section{Discussion}

Psoralens are synthesized naturally or synthetically. 8-methoxypsoralen (8-MOP) is commonly available oral and topical formulation in India. Psoralen coalesce with epidermal DNA which diminishes DNA replication thus ceases cell cycle. Psoralens and UV radiation also stimulate melanogenesis. In vitiligo, the mode of pigment induction of PUVA is hypothetical. The photoconjugation of psoralens in melanocyte DNA leads to mitosis, replication and proliferation of melanocytes, increased number of melanosomes and their further transfer to keratinocytes. Stimulation of cyclic adenosine monophosphate (cAMP) activity by
PUVA leads to increased synthesis of tyrosine. PUVA also affects immunological processes and may induce a suppressor T cell population and release IL-10 which is important for differentiation and activation of $\mathrm{T}$ regulatory cells that may suppress the auto-immune stimulus responsible for melanocyte destruction. PUVA also induces basic fibroblast growth factor (bFGF) and hepatocyte growth factor, which may aid in regrowth and migration of follicular melanocytes to the basal layer of skin. ${ }^{5,6}$

Vitiligo is an auto inflammatory idiopathic skin condition affecting $1 \%$ population, with symptoms of depigmented macules and patches. ${ }^{1}$ The present study was conducted to evaluate the efficacy of therapeutic 
effect of oral psoralen followed by PUVA therapy for vitiligo cases. A total 36 cases with acral, acrofacial and generalised vitiligo with more than $20 \%$ spread over body were considered.

Among the cases, generalised vitiligo is seen in $66.6 \%$ cases, acral was seen in $16.6 \%$ cases, acrofacial was seen in $16.6 \%$ cases. No cases with segmental and focal vitiligo in this study (Table 1). Study by Serkan Yazici et al, stated generalised vitiligo (16 cases) was common subtype followed by acrofacial (6 cases) and focal vitiligo (4 cases). ${ }^{7}$ Study by Hari Kishan Kumar Y et al, stated that $59.3 \%$ cases had generalized vitiligo, $30 \%$ cases had acral/acrofacial type, 5.3\% had focal and $5.3 \%$ had segmental type of vitiligo. ${ }^{8}$

In the present study erythema was the common side effect followed by xerosis, pruritus, nausea and vomiting, bulla and burning (Table 2). Serkan Yazici et al, in his study not observed any side effects except in one case who had reversible erythema. ${ }^{7}$ Hari Kishan Kumar $\mathrm{Y}$ et al, in his study stated that $7 \%$ cases reported mild erythema, burning, pruritus and $6 \%$ cases complained of xerosis. ${ }^{8}$

Due to PUVA therapy, in generalised vitiligo, marked response was found in $25 \%$ cases, good response in $45.8 \%$ cases, moderate response in $16.6 \%$ cases and mild response in $12.5 \%$ cases. In acral group, good response was seen in 50\% cases and moderate response was seen in 50\% cases. In acrofacial group, good response was seen in 50\% cases, moderate response was seen in $33.3 \%$ cases and mild response was seen in $16.6 \%$ case (Table 3 ).

In this study, the mean age of the patients was 37.5 years, mean duration of disease was 98.40 months and the mean percentage of area affected with vitiligo is $31.36 \mathrm{cms}$. The mean duration of treatment was 14.52 months with mean no of sessions is 62.18. In this study, the mean cumulative dose require to get marked response was $230.4 \pm 126.34 \mathrm{~J} / \mathrm{cm}^{2}$ (Table 4 ). Study by Hari Kishan Kumar Y et al, stated that lesser number of exposures (51.91) and lesser cumulative dose (46.8 \pm $25.2 \mathrm{~J} / \mathrm{cm}^{2}$ ) was enough to achieve $25-75 \%$ repigmentation (8). Study by Njoo et al, and other western studies noticed same repigmentation with more number of exposures $(76.3 \pm 16.7)(9-13)$. The results of present study is correlating with the other Indian studies which stating that Fitzpatrick type IV and V needs lesser number of exposures than Fitzpatrick type I and II (14-16).

\section{Conclusion}

PUVA therapy is a safe, effective and gold standard therapeutic modality for the treatment of vitiligo with minimal side effects. Which has a very good safety profile in Indian skin and have not been reported other side effects and cases of carcinogenesis. PUVA is better than UVB because of deep penetration. In this study, generalized type was best to respond well for the treatment than acral and acrofacial which are resistant. Erythema was the common side effect followed by xerosis, pruritus, nausea and vomiting, bulla and burning.

\section{References}

1. Dutta AK, Dhar S. Vitiligo: Past and present. Indian J Dermatol. 2002;47(3):132-40.

2. Stephen O. Kovacs, continuing medical education vitiligo. J Am Acad. Dermatol. 1998;38:647-66.

3. Noor SM, Khurshid K, Mehmood T, Haroon TS. Quality of life in vitiligo patients. $J$ Pak Assoc Dermatol. 2004; 14:55-8.

4. Halder RM. Childhood vitiligo. Clin Dermatol. 1997;15:899- 906.

5. Falabella R, Barona MI. Update on skin repigmentation therapies in vitiligo. Pigment Cell Melanoma Res. 2008;22:4265.

6. Wu CS, Lan CC, Wang LF, Chen GS, Wu CS, Yu HS. Effects of psoralen plus ultraviolet A irradiation on cultured epidermal cells in vitro and patients with vitiligo in vivo. Br J Dermatol. 2007;156:122- 9.

7. Serkan Yazici, Berrin Gunay, Emel Bulbul Baskan, Kenan Aydogan, Hayriye Saricaoglu, Şukran Tunali. The efficacy of narrowband UVB treatment in pediatric vitiligo: a retrospective analysis of 26 cases. Turk J Med Sci. 2017;47:381-384.

8. Y. Hari Kishan Kumar, G. Raghu Rama Rao, K. V. T. Gopal, G. Shanti, K. Veerabhadra Rao. Evaluation of narrow-band UVB phototherapy in 150 patients with vitiligo. Indian J Dermatol Venereol Leprol. 2009;75(2):162-166.

9. Scherschun L, Kim JJ, Lim HW. Narrow-band ultraviolet $\mathrm{B}$ is a useful and well-tolerated treatment for vitiligo. $J$ Am Acad Dermatol. 2001;44:999-1003.

10. Yones SS, Palmer RA, Garibaldinos TM, Hawk JLM. Randomized Double-blind trial of Treatment of Vitiligo: Efficacy of psoralen-UVA therapy vs narrowband-UV-B therapy. Arch Dermatol. 2007;143:578-83.

11. Njoo MD, Bos JD, Westerhof W. Treatment of generalized vitiligo in children with narrow-band (TL-01) UVB radiation therapy. $J$ Am Acad Dermatol. 2000;42:245-53.

12. Anbar TS, Westerhof W, Rahman AT, Khayyat MA. Evaluation of the effects of NB-UVB in both segmental and nonsegmental vitiligo affecting different body sites. Photodermatol Photoimmunol Photomed. 2006;22:157.

13. Gambichler T, Breuckmann F, Boms S, Altmeyer P, Kreuter A. Narrowband UVB phototherapy in skin conditions beyond psoriasis. J Am Acad Dermatol. 2005;52:660-70.

14. Kanwar AJ, Dogra S, Prasad D, Kumar B. Narrow-band UVB for the treatment of vitiligo: An emerging effective and welltolerated therapy. Int J Dermatol. 2005;44:57-60.

15. Dogra S, Prasad D. Combination of narrow band UVB and topical calcipotriene in vitiligo. Arch Dermatol. 2003;139:393.

16. Kanwar AJ, Dogra S. Narrow-band UVB for the treatment of generalized vitiligo in children. Clin Exp Dermatol. 2005;30:332-6.

How to cite this article: Narayana KB. Evaluation of the therapeutic effect of PUVA therapy in chronic vitiligo patients. Ind J Clin Exp Dermatol. 2018;4(3):237240. 